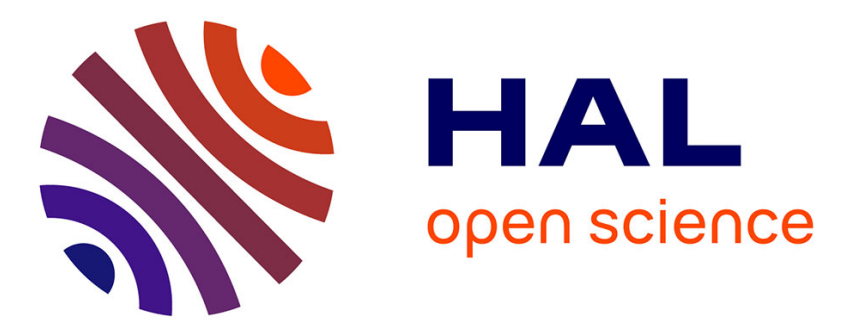

\title{
An Energy Efficient Smartphone Sensors' Data Fusion for High Rate Position Sampling Demands
}

Esubalew Alemneh, Sidi-Mohammed Senouci, Philippe Brunet

\section{To cite this version:}

Esubalew Alemneh, Sidi-Mohammed Senouci, Philippe Brunet. An Energy Efficient Smartphone Sensors' Data Fusion for High Rate Position Sampling Demands. 2019 16th IEEE Annual Consumer Communications \& Networking Conference (CCNC), Jan 2019, Las Vegas, United States. pp.1-6, 10.1109/CCNC.2019.8651755 . hal-02864526

\section{HAL Id: hal-02864526 \\ https://hal.science/hal-02864526}

Submitted on 18 Jan 2022

HAL is a multi-disciplinary open access archive for the deposit and dissemination of scientific research documents, whether they are published or not. The documents may come from teaching and research institutions in France or abroad, or from public or private research centers.
L'archive ouverte pluridisciplinaire HAL, est destinée au dépôt et à la diffusion de documents scientifiques de niveau recherche, publiés ou non, émanant des établissements d'enseignement et de recherche français ou étrangers, des laboratoires publics ou privés.

\section{다)(1) $(5$}

Distributed under a Creative Commons Attribution - NonCommerciall 4.0 International 


\title{
An Energy Efficient Smartphone Sensors’ Data Fusion for High Rate Position Sampling Demands
}

\author{
Esubalew Alemneh ${ }^{1,2}$, Sidi-Mohammed Senouci ${ }^{1}$, Philippe Brunet ${ }^{1}$ \\ ${ }^{1}$ DRIVE EA1859, Univ. Bourgogne Franche-Comté, F58000, Nevers, France \\ ${ }^{2}$ Bahir Dar University, Bahir Dar Institute of Technology, Faculty of Computing, P.O.Box 26, Bahir Dar, Ethiopia \\ Esubalew_Jalew@etu.u-bourgogne.fr,Sidi-Mohammed.Senouci@u-bourgogne.fr, Philippe.Brunet@u-bourgogne.fr
}

\begin{abstract}
Many smartphone-based traffic safety applications have been proposed in literatures. These applications demand very high position sampling to safeguard vulnerable road users. European Telecommunication Standards Institute (ETSI) has defined time interval between Cooperative Awareness Messages for collision risk warning to be between 1s and 0.1s. This implies that geographical awareness information has to be sampled between the frequencies $1 \mathrm{~Hz}$ and $10 \mathrm{~Hz}$ inclusive. However, an investigation we made depicts that current smartphones can't support such high rate location sampling. Even though they meet the aforementioned sampling requirements, high rate sampling of position data is an energy hungry process. In light of this, we have proposed an energy efficient position prediction method that fuses GPS and Inertial Navigation Systems (INS) sensors data to estimate pedestrians' positions at high rate. INS based dead reckoning is performed to extrapolate positions from last known location when GPS reading is unavailable and when GPS fix is realized the reading is used to correct dead reckoning parameters in addition to serving as a location fix. The proposed solution is compared to a position prediction method that relies solely on GPS data on two selected pedestrian trajectories. The result demonstrates that fusing GPS and INS position data has an average improvement of $30 \%$ and $61.4 \%$ in error in distance and direction respectively. The proposed position prediction algorithm is also applied to sensors data that are obtained by relaxing sampling rates with the objective of sparing smartphone's energy. In this regard, first energy efficiency of different position sampling rates of GPS and INS sensors are evaluated and then the algorithm is applied to the sampling frequencies that are proven to husband energy. The outcome of the evaluation is that the battery life of smartphones can be doubled by compromising accuracy of estimated distance and direction by only $11.5 \%$ on average.
\end{abstract}

Keywords—inertial navigation systems, GPS, traffic safety, pedestrian navigation, dead reckoning

\section{INTRODUCTION}

Smartphones have become part of everyday life of human beings. Gaming, navigation, monitoring environmental and traffic conditions, supervising health of possessors, intervention on our behalf, and social networking are some of their applications [1] [2]. In recent days, due to their wide existence and increased storage and computation capability smartphones have become important ingredients of infrastructure-less traffic safety, infotainment and traffic efficiency applications. A staggering increase in their capability is further enhanced by their sensing potential which is another feature that makes the devices more intelligent and essential part of life.

Though the number of sensors may vary from one smartphone to the other, typical smartphone contains accelerometer, a digital compass, a gyroscope, a GPS, quad microphones, dual cameras, near-field communication, a barometer, light, proximity, and temperature sensors [2]. However, smartphones are far from becoming apotheosis sensing devices. Producers of the devices purposefully make them compact and small so that they will be cheaper and fulfill user preferences. Size reduction and subjection of the devices to non-ideal environments compromise the sensor accuracy and reliability highly and this has become hindrance to fulfil application requirements. For instance, ETSI has enacted maximum and minimum time interval between beacon generations to be $1 \mathrm{~s}$ and $0.1 \mathrm{~s}$ for collision risk warning systems. Despite the fact that smartphone-based solutions are proposed for traffic safety by warning about-to-occur collisions based on positions of vehicles and vulnerable road users (VRU) [3][4], present smartphones' position sampling rate do not meet the minimum time interval positions have to be sampled.

A research conducted by Zhizhong et al. in 2013 [5] has indicated that maximum GPS sampling rates of smartphones is 1s [5]. Our investigation on maximum sampling rate of latest smartphone showed that things haven't changed till now. Losses in connections and imperfect behavior of the data collection application further increase the sparseness of the data [6]. From these facts we can conclude that location updates of smartphones do not meet applications requirements since pedestrians with maximum $1.83 \mathrm{~m} / \mathrm{s}$ walking and $4.2 \mathrm{~m} / \mathrm{s}$ running speeds [7] could face traffic accident in the time interval between two consecutive locations updates as vehicles impact velocity could reach up to $80 \mathrm{~m} / \mathrm{s}$ [8]. Therefore, to celebrate smartphones' involvement for traffic safety we have proposed a solution that uses inertial sensors to predict positions in case of unavailability of GPS location fixes. The method integrates GPS and low-cost inertial sensors to satisfy high position sampling demand of traffic safety applications.

INS can be used to pinpoint position of smartphone holders and they are not dependent on any external measurements like radios [9]. It is possible to extrapolate locations from reference points by calculating distance travelled and direction of movement from inertial sensors data. INS sensors data can be sampled at different rates. High sampling rates of GPS and INS sensors is known to drain smartphone batteries rapidly. Therefore, applications that demand fast position sampling must address the issue of energy consumption in order not exacerbate energy hunger of smartphones. This can be done by slacked sampling of GPS and INS sensors data instead of sampling them at their fastest possible time. However, we have to take a caution not to harm accuracy of position prediction by reducing the number of data points for estimation. Our work in this research includes evaluating energy efficiency of various GPS and INS sensors data sampling rates and application of the prediction algorithm proposed for sampling rates that are found to increase energy efficiency of smartphones. 
This paper reports an energy efficient dead reckoning method to estimate locations of VRUs particularly pedestrians. The locations are estimated at required rate from known location and kinematics of the pedestrian by integrating GPS and INS sensors data. The contributions of the research are;

- We have conducted an experiment to determine if different location methods endorsed by present smartphones fulfil high location sampling requirements of traffic safety applications. The experiment shows that maximum sampling rates of current smartphones' location methods do not meet application requirement of traffic safety applications.

- In order to cope with high location sampling rate demands of traffic safety applications, we have proposed a novel INS and GPS sensors data fusion method to track pedestrians using their smartphones. The method doesn't depend on map information or movement history of VRU. The evaluation of the solution using two selected trajectories shows that it is able to improve accuracy of position prediction that solely depends on GPS.

- Energy consumption of various GPS and INS sampling rates are experimentally investigated. The proposed position prediction method is applied to sampling rates which contributes to smartphones energy efficiency. The result shows that with small compromise of accuracy large amount of energy can be gained by relaxing sampling period of INS sensors.

The rest of the paper is organized as follows. Section 2 summarizes related works in INS based dead reckoning and energy efficiency. An investigation conducted to verify maximum sampling rate of location methods is presented in Section 3. Section 4 discourses detail of the proposed solution while Section 5 is dedicated to discussions of the evaluation results made on the proposed algorithm and energy efficiency of different GPS and INS data sampling rates. Finally, conclusion is presented in Section 6 .

\section{LITERATURE REVIEW}

Traffic safety applications necessitate high rate position sampling to safeguard pedestrians and other VRUs from traffic accidents. Though smartphones have been recommended for traffic safety applications as sources of positions of VRUs [34] [10-11], their position sampling rate is limited. Therefore, at the time GPS fix is not possible position of VRUs must be dead reckoned using supplementary positioning systems. Dead reckoning (DR) can be applied in offline mode [12] or online mode [13]. Offline DR is used for post-processing of locations after collecting known locations. Online DR is suitable for realtime systems where estimation has to be made immediately after a known location is captured. Most position estimation solutions proposed are either for vehicular navigation [13-15] or for indoor pedestrian navigation [12] [16-17]. In order to extrapolates next positions from known GPS locations for pedestrian traffic safety, an online DR that can be applied in indoor or outdoor environments is required.

Energy saving by lowering sensor data sampling, absence of GPS location signals, and a need to improve location accuracy are some of the reasons for applying position prediction methods in navigation. Outdoor navigation systems that are used in open sky scenarios can use GPS based locations.
However, to reduce smartphone energy consumption by lowering GPS sampling rate, GPS based localizations can be supplemented by dead reckoning that involves low energy cost sensors [12-13] [18]. INS sensors helps to improve positioning reliability in situations where GPS location is not available due to obstruction of GPS satellites by high buildings, trees etc. INS can be also integrated with GPS for best location accuracy [14] as both methods suffer from their own drawbacks. According to Zengshan Tian et al. in [19] navigation information obtained from the INS sensors is boasted with continuity, high dataupdating rate, good short-term accuracy, and stability. In this research INS sensors are used to supplement GPS based positioning to fulfill higher location sampling rate requirements than the current smartphones can support. As our experiment has proved and other researchers have indicated [5] maximum GPS sampling rate is $1 \mathrm{~Hz}$ which is below the minimum sampling frequency enacted by ETSI.

To make position predictions, distance travelled and direction of movement have to be estimated. Most dead reckoning solutions use accelerometer to predict displacement by first detecting step events and estimating stride length [16] [20-21]. Direction of movement is usually estimated from magnetometer and gyroscope data and position prediction is made after every stride or more. For problems that require very fast position predictions multiple estimations must be made in a period of one stride. Therefore, step detection and stride length estimation-based position prediction do not fit for such application. Hence, the proposed prediction method involves estimation of displacement by using conventional kinematic equations of distance and velocity. GPS readings are used to correct dead reckoning parameters periodically in addition to serving as correct positions of pedestrian trajectory. Accelerometer and magnetometer are used to estimate attitude of movement of pedestrian. Intermediate positions estimated from displacement and heading are set to pass through Kalman Filter (KF) for further improvement of the positions.

Gathering GPS and INS sensor data at high data-updating rates is an energy hungry process [5] [22-25]. [22-23] have shown sampling GPS at different sampling periods has different energy consumption. According to [5] [24] normal INS data sampling took $25 \%$ less battery power than fastest sampling. We have studied how much energy hungry different GPS and INS data samplings are at a very small sampling period. The application of position prediction method proposed on sensor data obtained by energy efficient sampling periods demonstrate that significant amount of energy can be saved with small effect on accuracy of prediction. The following section presents an experiment conducted to check maximum sampling rate of current smartphones.

\section{MAXIMUM GPS SAMPLING RATE OF SMARTPHONES}

Maximum GPS sampling rate of smartphones depend on factors like used location method, make of the phone, embedded GPS chipset, as well as version and type of operating system of the mobile device. For collision risk warning system ETSI enacted maximum and minimum time interval between beacon generations to $1 \mathrm{~s}$ and $0.1 \mathrm{~s}$, respectively [26]. This implies that location sampling frequency should be between $1 \mathrm{~Hz}$ and $10 \mathrm{~Hz}$ since the beacons comprises of location information. To check if modern smartphones meet high GPS sampling requirements of collision risk warning systems we have conducted an experiment. The location methods supported by current 
smartphones are high accuracy, GPS only, and network (Wi-Fi a Mobile Internet). High accuracy location method, which is also termed Assisted-GPS (A-GPS), uses GPS chip and networks to get location fixes. GPS only location method uses solely GPS chips and network location methods may depend on Wi-Fi or mobile data internet connection.

All applications except our experimental application were denied location access and the mobile device was set to run only the default manufacturer processes. Samsung smartphone (Model-SM-A520F) was used exclusively for this controlled experiment. An Android application is developed which can read smartphone location at the highest possible rate. By enabling each location methods turn by turn the experiments were conducted in indoor and outdoor environments. Keeping the smartphone with the application running on front jacket pocket one of the researchers walked for about an hour for each experiment. The position data is saved in a log file. The average minimum possible sampling period is presented in TABLE 1 .

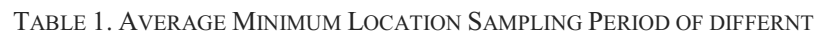
LOCATION METHODS

\begin{tabular}{|l|l|}
\hline Location Method & Sampling Period (ms) \\
\hline GPS only & 1003.84 \\
\hline A-GPS & 1281.28 \\
\hline Mobile Internet & 20521.42 \\
\hline Wi-Fi & 20178.13 \\
\hline
\end{tabular}

The highest average GPS fix frequency is achieved by GPS only location system. Using this location method, we can get location updates approximately each second. This value confirms with the result obtained in [5]. Location obtained from mobile internet and Wi-Fi based location methods' is too sparse for traffic safety applications. A-GPS sampling rate is lower than GPS only based location method as it uses network location methods when GPS fixes are not possible. The experiment reveals that GPS sampling rates of current smartphones do not satisfy application requirement of traffic collision risk warning systems. Thus, location methods that can provide positions at higher rates should work together with GPS based location to meet high rate location demand of traffic safety applications. The next section is dedicated to describe details of our solution that enables high frequency positioning.

\section{POSITION PREDICTION SYSTEM}

Position prediction system proposed is aimed to extrapolate locations in a very short time interval $(100 \mathrm{~ms})$. A GPS fix gained every second is used as correct location and to adjust dead reckoning parameters for forthcoming predictions. If no new location is received position estimation is made using INS sensors data. Components of the system are shown in Fig.1. Each component is elaborated in subsequent sub-sections.

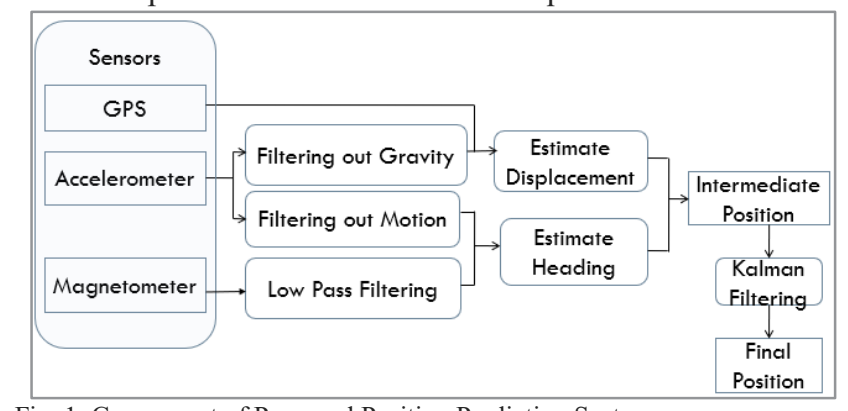

Fig. 1. Component of Proposed Position Prediction System

\section{A. Sensors and Sensor Data Processing}

Accelerometer and Magnetometer are two INS sensors used for the position predictions in addition to GPS sensor. Speed from GPS readings is used to estimate displacement by adjusting velocity of pedestrians obtained from accelerometer. Raw acceleration data is split into two by filtering out gravity. This process results in linear acceleration and gravity virtual sensors. The two software-based sensors are synthesized through a combination of accelerometer and gyroscope inputs and Android performs optimized filtering and sensor fusion processes by applying Extended KF [27]. Magnetometer and gravity sensors are employed to predict movement direction of smartphone holder. As magnetometer is susceptible to working machines and metals in the vicinity to remove the highfrequency noise from its output, a low-pass filtering has been applied. After repeated experiments, we found that at cutoff frequency $\mathrm{f}=0.01 \mathrm{~Hz}$ the heading result is more durable and accurate in the environment the test is conducted.

\section{B. Position Prediction}

After pre-processing of sensor data, position is estimated by figuring out displacement and heading of the pedestrian [16] [19]. Linear acceleration is used together with speed obtained from GPS to estimate displacement. Heading is computed from accelerometer without motion component and corrected magnetometer values. Once the two values are available, intermediate position can be calculated using equation (1).

$$
\begin{aligned}
& X=X_{0}+D \sin (\beta) \\
& Y=Y_{0}+D \cos (\beta)
\end{aligned}
$$

Where, $\left(\mathrm{X}_{0}, \mathrm{Y}_{0}\right)$ and $(\mathrm{X}, \mathrm{Y})$ are initial and intermediate positions, $\mathrm{D}$ is the displacement and $\beta$ is the heading. The destination position is obtained by passing intermediate position throw KF to improve accuracy of prediction. KF equations presented in [28] are used since the method have improved GPS accuracy by $12.59 \%$. The detail of calculation of displacement and heading are presented beneath.

\section{Displacement Calculation}

Calculating displacement using inertial based pedestrian dead reckoning usually involves step detection and step length estimation [17] [19]. This method is not applicable if position estimation has to be made in a very short time interval since many predictions may be needed in a period of one stride which is $0.4 \mathrm{~m}$ to $1.0 \mathrm{~m}$ in length [16]. This is true for the application in consideration as position prediction has to be made every $0.1 \mathrm{~s}$. Conventional kinematics equations shown on equation (2) are used to calculate displacement.

$$
\begin{aligned}
& \mathrm{V}_{\mathrm{t}}=\mathrm{V}_{0}+\mathrm{A}_{\mathrm{t}} * \mathrm{t} \\
& \mathrm{D}_{\mathrm{t}}=\mathrm{V}_{\mathrm{t}} * \mathrm{t}+0.5 * \mathrm{~A}_{\mathrm{t}} * \mathrm{t}^{2}
\end{aligned}
$$

Where, $D_{t}, V_{t}$, and $A_{t}$ are displacement, velocity and acceleration at time $t, t$ is change in time, $V_{0}$ is the initial velocity. $\mathrm{V}_{0}$ is set to 0 at the beginning. Velocity is calculated by integration of acceleration found from accelerometer without gravity. Since acceleration and velocity take vector values, integration is made for each axis. Due to accumulated error velocity extracted from linear acceleration tends to increase exponentially over a short period of time, see Fig.2. Therefore, velocity from GPS fixes is fused and used as initial velocity at each GPS fix. Let $V^{\text {gps }}$ be speed obtained from GPS, and $\beta$ be heading, then $X$ and $Y$ components of initial velocities at ground truth point are computed using equation (3) [16]. 


$$
\begin{aligned}
& \mathrm{V}_{0 \mathrm{x}}=\mathrm{V}^{\mathrm{gps}} \sin (\beta) \\
& \mathrm{V}_{0 \mathrm{y}}=\mathrm{V}^{\mathrm{gps}} \cos (\beta)
\end{aligned}
$$

We can't use only the velocity extracted from GPS fixes as it wouldn't capture frequent changes as GPS are sampled every one second and displacement is calculated every $0.1 \mathrm{~s}$.

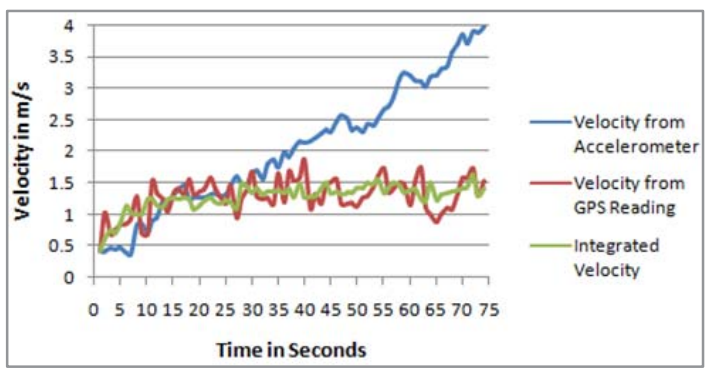

Fig. 2. Velocities from Accelerometer and GPS Readings and Fusion of the two. Integrated velocity is more stable in comparison with velocity from accelerometer. By taking this velocity the distance between two GPS fixes is found to be approximately the same as sum of distances between GPS points.

\section{Heading Estimation}

Heading is the deviation of the smartphones $\mathrm{Y}$-axis from magnetic north, measured clockwise in the East-North plane. Corrected values from magnetometer and gravity portion of accelerometer are integrated to estimate attitude angle of the smartphone [17]. From the two set of values a rotation matrix is produced to map points from local coordinate system (LCS) to global coordinate system (GCS) to resolve random placement of smartphones. GCS is a real-world North-East-Gravity direction coordinate system. If $\mathrm{R}_{\mathrm{t}}$ is the rotation matrix and $\gamma$ is an azimuth which is degrees east of magnetic north in LCS, then the orientation is converted to GCS using equation (4).

$$
\gamma^{\mathrm{GCS}}=\mathrm{R}_{\mathrm{t}} \gamma^{\mathrm{LCS}}
$$

The actual heading of a user is forecasted by accounting declination angle. Refer [12] [17] to get detail on heading estimation. Fig. 3 displays comparison of heading calculated using INS sensors and GPS bearing during a short trip in the test site. The test data which is collected from rectangular trajectory depicts that the two values are similar with only minor differences.

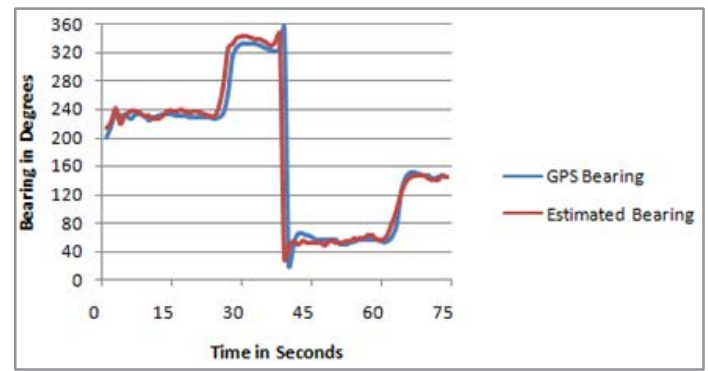

Fig.3.

Comparison of Heading Estimation with Ground Truth GPS Bearing

\section{V.EVALUATIONS AND RESULTS ANALYSIS}

In this section evaluation result of the proposed method is presented, energy efficiency of various GPS and INS sampling rates are assessed, the proposed method is applied to energy efficient sampling methods and the results are discussed.

\section{A. Evaluation of the Proposed Method}

The proposed method is tested with a data collected using Android application that reads GPS points at the highest possible sampling period. The algorithm running on the smartphone calculates the displacement and heading angle and estimates locations at $0.1 \mathrm{~s}$ interval. Accelerometer and magnetometer were set to sample at maximum rate and average values are taken for the estimation period. Ground truth data is collected using real-time kinematics (RTK) position device (AsteRx4 OEM). Two trajectories are conceived to test the proposed system. The first trajectory is a straight path with only one turn while the second is a rectangular path. The proposed GPS and INS Sensor based Prediction (GISP) is compared in terms of error in distance of predicted points and deviation of heading from ground truth points of walking path with Simple Linear Prediction (SLP) that uses only GPS data. The method utilizes speed and bearing of the most recent GPS fix to estimate the next position.

Fig. 4 depicts average and maximum distance errors of predicted points from ground truth points for the two prediction methods on the two trajectories. Maximum prediction distance error is included to show the worst-case inaccuracies of prediction algorithms. For both trajectories the method that integrates GPS and INS sensors data has lesser errors than the one that uses only GPS data. In fact, integrating inertial sensors with GPS improved position prediction accuracy by about $30 \%$ percent since direction of movement is updated frequently from INS sensors. The prediction is more accurate for Trajectory 1 due to its simplicity.

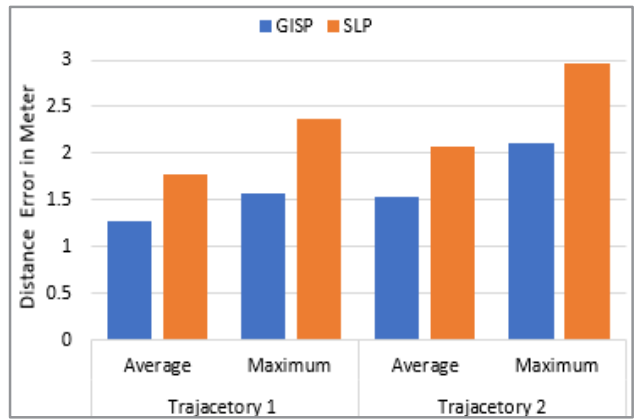

Fig.4. Average and Maximum Error in Distance of Predicted Points for the two Trajectories.

Fig. 5 shows average error in heading of the two methods. Error in the second trajectory is higher than the first due to more turns in the path. In GISP heading of the pedestrian at each prediction point is feed from INS sensors. Because of this, the method improves accuracy of heading estimation by about $61.4 \%$ from the one which relies only on GPS readings. Because of space limitation we can't include visualization of the prediction in this report.

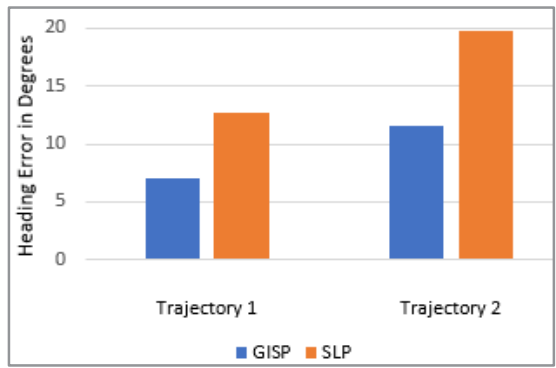

Fig. 5. Error in Heading of GISP and SLP for the Two Trajectories 


\section{B. Sampling Rates and Energy Consumption}

Maximum sampling period of current smartphones are found to be one second. Higher sampling periods however could be used for energy saving as long as noticeable energy saving can be achieved and accurate prediction can be made. Energy consumption of GPS sampling at one, two, three and four seconds is evaluated. Relaxed sampling periods of INS sensor data can also help in saving energy. Two default Android and one user defined inertial sensor sampling rates are assessed. The details of inertial sensor sampling types considered are displayed in TABLE 2.

TABLE 2. ANDROID INS SAMPLING TYPES

\begin{tabular}{|l|c|c|c|}
\hline & FASTEST & GAME & USER DEFIND \\
\hline $\begin{array}{l}\text { Average Sampling } \\
\text { Period (ms) }\end{array}$ & 5 & 20 & 50 \\
\hline $\begin{array}{l}\text { Average Number } \\
\text { of Samples }\end{array}$ & 20 & 5 & 2 \\
\hline
\end{tabular}

Position prediction method explained in previous sections samples GPS and INS data at highest possible frequencies. Sampling frequency of GPS and INS affect energy consumption of smartphones and accuracy of position predictions [5] [2224]. The higher sensor data sampling rate implies the higher energy consumption and the more accurate position estimation. In this section energy consumption of various GPS and INS sampling rates are assessed. Next, prediction exactitude of prediction method proposed using various sampling methods that are found to enable obtrusive energy saving are evaluated.

Three Android applications are developed to collect energy consumption information of the smartphone used for the experiment. Application1 is used to collect battery information of a phone that involve sampling neither GPS nor INS. Application2 collects battery detail of a smartphone that samples GPS at 1, 2, 3 and 4 seconds. Application3 is used to get battery information of a phone that samples GPS each second and INS at rates specified in TABLE 2. A smartphone with battery capacity of $3000 \mathrm{mAh}$ is used for the experiment. The following configurations are made before conducting the experiment. The smartphone is reset to factory setting. During testing no application runs except Application1, Application2 or Application3 and the smartphone is used strictly for this experiment. All types of connections are turned off and screen brightness is set to the lowest possible value. Root access of the android smartphone is obtained so as to read current discharge of the smartphone. The smartphone is charged to $100 \%$ before each experiment. For each experiment the smartphone holder walked for 50 minutes in outdoor and indoor walking paths. For additional 10 minutes the smartphone is placed on a flat desk in office.

The result of energy consumption of smartphones at different GPS Sampling period and INS Sampling types is indicated in Fig. 6. GPS reading at GPS sampling periods considered has no significant energy consumption difference. As stated in [22-23] location updates at lower intervals have very subtle differences in the battery decay over time. In onehour test, battery level of the smartphone has decreased from $100 \%$ to $97.17 \%$ in $1 \mathrm{~s}$ GPS sampling period and from $100 \%$ to $97.84 \%$ in 4 s GPS sampling period. The drop of battery level due to GPS sampling in our experiment in general is modest since the smartphone is used solely for this purpose and it has high battery capacity. Fastest INS sampling consumes energy quicker than the other two sampling types. A battery that could have stayed for 23 hours with GAME and 26 hours with USER DEFINED INS sampling types will run out only in 11 hours.

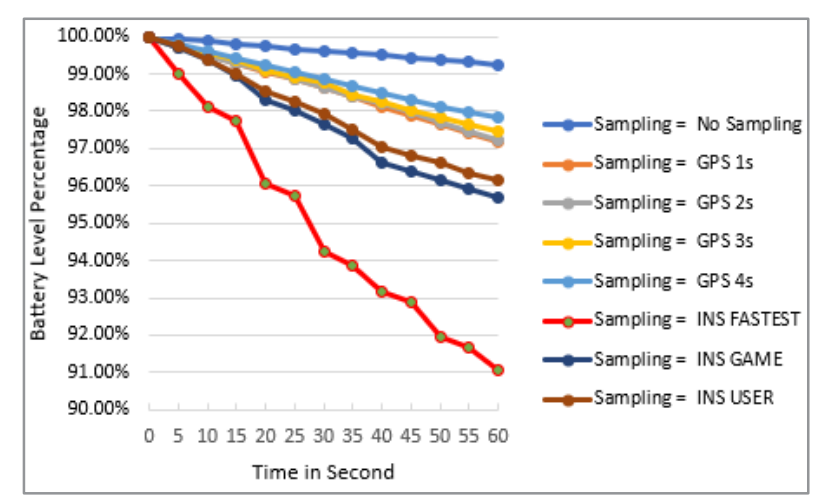

Fig.6. Energy Consumption of Different GPS and INS Sampling Rates

\section{Sampling Rates and Prediction Accuracy}

Small-scale energy overhead on different GPS data sampling period doesn't convince us to compromise accuracy by choosing higher GPS sampling periods. In case of INS data sampling on the other hand a noticeable energy saving can be achieved by selecting energy efficient INS data sampling type. Accuracy of position prediction depends on whether the prediction is made on straight or on curved trajectories. Hence, the evaluation is made for straight and curved sections of Trajectory 2. From the rectangular trajectory three curves and four straight lines are considered. In addition to GISP and SLP, predictions that involve GAME (viz GISP_GAME) and USER DEFINED (viz GISP_USER) inertial sensor sampling are compared.

Average error in distance and attitude angle of predicted positions for straight and curved trajectories is displayed in Fig. 7 and 8, respectively. GISP is the most accurate prediction method on both straight and curved trajectories since it takes many INS data points to make the prediction. On straight line there is no abrupt change of direction and this is the reason why SLP performs well. In curved trajectories SLP performs worst because of lack of updated movement direction until the next GPS fix is attained. GISP_USER do not work well on straight and curved roads since the number of inertial sensors data taken on average are two and sometimes the prediction may be made using only one or no data points. Unless we take considerable number of sensor data, due to noisy nature of inertial sensors prediction is poor. Prediction accuracy of GISP_GAME is the second best to GISP. Sampling period of this prediction is lower than GISP but higher than GISP_USER.

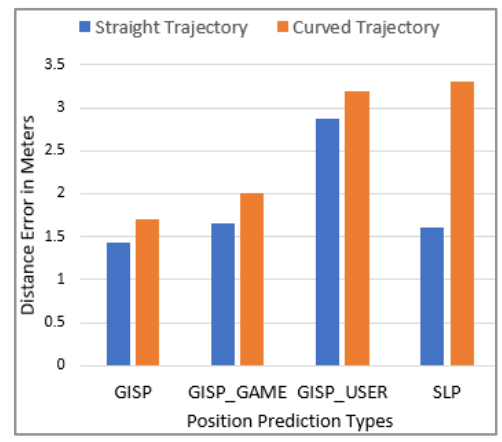

Fig.7. Average Error in Distance of Different Position Prediction Types 


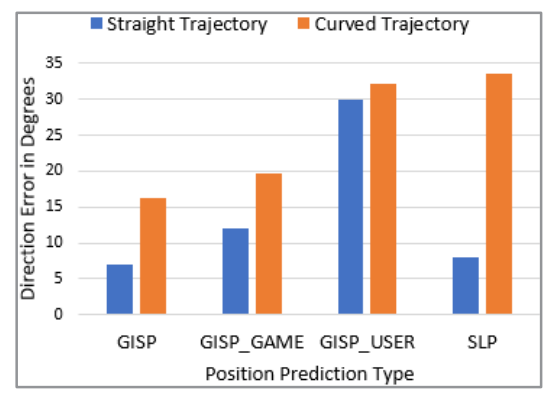

Fig.8. Average Error in Movement Direction of Pedestrian in Different Position Prediction Types

GISP_GAME is recommended position prediction method when pedestrians are in non-traffic risky regions since it doubles the life of battery with $11.5 \%$ compromise of accuracy.

\section{CONCLUSION}

Even though smartphones have become integral part of traffic safety applications, they do not support high position sampling requirement of the applications. Therefore, we have proposed inertial navigation system-assisted positioning system that works together with GPS sensors to predict locations of pedestrians at a very high rate. The method uses GPS fixes to correct dead reckoning parameters and as ground truth points for the next position estimations. A method that uses only GPS readings to extrapolate positions is compared with the new method using data from two trajectories conceived. As the proposed system integrates inertial sensors that have very high short-term accuracy and GPS which has very good long-term accuracy it outperforms GPS only based prediction. So as to make the position prediction energy conserving, energy efficiency of various data sampling rates of GPS and INS data sampling are investigated. Results show that by sparsening GPS samples the energy that could be saved is minimal while accuracy could be highly affected. However, by reducing sampling frequency of INS data sampling obtrusive energy is able to be saved with minor accuracy compromise. Therefore, inertial sensors can be integrated with GPS sensor in an energy efficient way to satisfy high position sampling rate demand of traffic safety applications.

\section{REFERENCES}

[1] F. Xia, C.-H. Hsu, X. Liu, H. Liu, F. Ding, and W. Zhang, "The power of smartphones," Multimedia Syst., vol. 21, no. 1, pp. 87-101, Feb. 2015.

[2] A. Campbell and T. Choudhury, "From Smart to Cognitive Phones," IEEE Pervasive Computing, vol. 11, no. 3, pp. 7-11, 2012.

[3] E. Alemneh, S.-M. Senouci, and P. Brunet, "PV-Alert: A fog-based architecture for safeguarding vulnerable road users," 2017 Global Information Infrastructure and Networking Symposium, 2017.

[4] U. Hernandez-Jayo, I. De-la-Iglesia, J. Perez, "V-Alert: Description and Validation of a Vulnerable Road User Alert System in the Framework of a Smart City", Sensors 15, Vol. 8, pp. 18480-18505, 2015.

[5] Z. Ma, Y. Qiao, B. Lee, and E. Fallon, "Experimental evaluation of mobile phone sensors," 24th IET Irish Signals and Systems Conference, 2013.

[6] A. Jaiswal, Y. Chiang, C. A. Knoblock, and L. Lan, "Location Prediction with Sparse GPS Data", In Proceedings of the 8th Geographic Information Science, 2014.

[7] H. Hamdane, T. Serre, C. Masson, and R. Anderson, "Issues and challenges for pedestrian active safety systems based on real world accidents," Accident Analysis \& Prevention, vol. 82, 2015.

[8] Y. Han, Q. Li, W. He, F. Wan, B. Wang and K. Mizuno, "Analysis of Vulnerable Road User Kinematics Before/During/After Vehicle Collisions Based on Video Records", IRCOBI Conference, Antwerp, Belgium, 13-15 September, 2017
[9] A. Correa, M. Barcelo, A. Morell and J. L. Vicario, "A Review of Pedestrian Indoor Positioning Systems for Mass Market Applications," Sensors, vol. 17, no. 8, p. 1927, 2017.

[10] K. David and A. Flach, "CAR-2-X and Pedestrian Safety," IEEE Vehicular Technology Magazine, vol. 5, no. 1, pp. 70-76, 2010.

[11] C. Sugimoto, Y. Nakamura, and T. Hashimoto, "Prototype of pedestrianto-vehicle communication system for the prevention of pedestrian accidents using both $3 \mathrm{G}$ wireless and WLAN communication," 3rd International Symposium on Wireless Pervasive Computing, 2008.

[12] M. Kourogi and T. Kurata, "A method of pedestrian dead reckoning for smartphones using frequency domain analysis on patterns of acceleration and angular velocity," IEEE/ION Position, Location and Navigation Symposium, 2014.

[13] K. Nawarathne, F. Zhao, F. C. Pereira, and J. Luo, "Dead reckoning on smartphones to reduce GPS usage," 13th International Conference on Control Automation Robotics \& Vision, 2014.

[14] Wei-Wen Kao, "Integration of GPS and dead-reckoning navigation systems," Vehicle Navigation and Information Systems Conference, 1991.

[15] J.-K. Liao, K.-W. Chiang, and Z.-M. Zhou, "The Performance Analysis of Smartphone-Based Pedestrian Dead Reckoning and Wireless Locating Technology for Indoor Navigation Application," Inventions, vol. 1, no. 4, p. 25, May 2016.

[16] W. Kang and Y. Han, "SmartPDR: Smartphone-Based Pedestrian Dead Reckoning for Indoor Localization,” IEEE Sensors Journal, vol. 15, no. 5, pp. 2906-2916, 2015.

[17] R. Zhou, "Pedestrian dead reckoning on smartphones with varying walking speed," 2016 IEEE International Conference on Communications, 2016.

[18] J. Biagioni, A. B. M. Musa, and J. Eriksson, "Thrifty tracking," Proceedings of the 21st ACM SIGSPATIAL International Conference on Advances in Geographic Information Systems - SIGSPATIAL13, 2013.

[19] Z. Tian, Y. Zhang, M. Zhou, and Y. Liu, "Pedestrian dead reckoning for MARG navigation using a smartphone," EURASIP Journal on Advances in Signal Processing, 2014.

[20] L. Ilkovičová, P. Kajánek, and A. Kopáčik. "Pedestrian Indoor Positioning and Tracking using Smartphone Sensors, Step Detection and Map Matching Algorithm." International Symposium on Engineering Geodesy. 2016.

[21] L. Ilkovičová, P. Kajánek, and A. Kopacik, "Pedestrian indoor positioning and tracking using smartphone sensors, step detection and map matching algorithm", SIG 2016 - International Symposium on Engineering Geodesy, 20-22 May 2016

[22] T. O. Oshin, S. Poslad, and A. Ma, "Improving the Energy-Efficiency of GPS Based Location Sensing Smartphone Applications," 2012 IEEE 11th International Conference on Trust, Security and Privacy in Computing and Communications, 2012.

[23] C.-M. Huang, C.-H. Lee, and W.-S. Chen, "A power efficient pedestrian touring scheme based on sensor-assisted positioning and prioritized caching for smart mobile devices," 201321 st International Conference on Software, Telecommunications and Computer Networks , 2013.

[24] T. Liu, C. Chen, M. King, G. Qian, and J. Fu, "Balancing Power Consumption and Data Analysis Accuracy Through Adjusting Sampling Rates: Seeking for the Optimal Configuration of Inertial Sensors for Power Wheelchair Users," Lecture Notes in Computer Science Digital Human Modeling. Applications in Health, Safety, Ergonomics and Risk Management: Ergonomics and Health, 2015.

[25] P.-L. Shih, P.-J. Chiu, Y.-C. Cheng, J.-Y. Lin, and C.-W. Yi, "EnergyAware Pedestrian Trajectory System," 41st International Conference on Parallel Processing Workshops, 2012.

[26] European Telecommunications Standards Institute, "Intelligent Transport Systems (ITS); Vehicular Communications; Basic Set of Applications; Definitions", ETSI, FRANCE, 2009

[27] android.com, "Sensor Types", 2017. [Online]. Available: https://source.android.com/devices/sensors/sensor-types\#gravity. [Accessed: 22-Mar-2018]

[28] E. Alemneh, S.-M. Senouci, and P. Brunet, An Online Time Warping based Map Matching for Vulnerable Road Users' Safety", 14th International Wireless Communications and Mobile Computing Conference, 2018. 